Anuj Sharma, Gagan Anand and Amneesh Singla

\title{
Ecological synthesis of $\mathrm{CuO}$ nanoparticles
}

ABSTRACT. The preparation of $\mathrm{CuO}$ nanoparticles with a mean primary particle size of $21 \mathrm{~nm}$ is described, using commercially available precursors. A key component of the method is sonochemical energy input. The overall method is energy-efficient with minimal waste and is therefore ecology (biosphere)-preserving. In liquid suspension the particles from agglomerates with a mean size of $c .500 \mathrm{~nm}$.

Keywords: copper oxide, cupric oxide, nanoparticles, p-type semiconductor, sonochemical method Nanotechnology Perceptions 14 (2018) 38-43

doi: 10.4024/N01SH18A.ntp.14.01 